\title{
Thomas Daiber
}

Justus-Liebig-Universität (Gießen, Deutschland)

\section{Die Strategie mündlicher Narrative und das kommunikative Gedächtnis}

\section{Strategie: intentionale Ordnung eines Themengebietes}

Es gehört zum sozialen Wesen des Menschen, sich mit anderen auszutauschen, Anschauungen zu versprachlichen und im Gespräch deren Validität zu verifizieren. Die kulturwissenschaftliche Theorie vom kommunikativen Gedächtnis (etwa Assmann 2000) beruht auf dem Phänomen des mündlichen Gesprächs und setzt dessen Funktion als alltagsweltlich bekannt voraus. Es ist aber auch eine Aufgabe der Textlinguistik, die Charakteristika kommunikativen Erinnerns an Produkten der konzeptionellen Mündlichkeit nachzuweisen.

Kommunikatives Erinnern besteht nicht in der expliziten intersubjektiven Aushandlung der Bewertung von Wirklichkeit. Zum einen wäre nicht klar, warum ein solches Aushandeln nicht einfach Debatte benannt würde, zum anderen wäre nicht klar, was dann der Erinnerungsgehalt genannt werden sollte. Die Debatte? Die einzelnen Standpunkte innerhalb der Debatte? Nur der Ausgang der Debatte (sofern sie einen hatte)? Das kommunikative Gedächtnis besitzt vielmehr eine Struktur, welche eine intersubjektive Gleichheit der Form des Erinnerns erzeugt. Diese Struktur kann «Rahmen» genannt werden:

Der Rahmen bezieht sich auf die Prämissen, nach denen die Situation bzw. das Ereignis zu deuten ist, und er ist «metakommunikativ» in dem Sinne, dass er «Anweisungen» darüber enthält, wie mögliche Ereignisse innerhalb des Rahmens zu verstehen sind (Schmitt 2009: 25).

Linguistisch korrespondiert der soziologischen Begrifflichkeit im weitesten Sinne der Begriff des Weltwissens bzw. das «Situationsmodell», also die im episodischen Gedächtnis gespeicherte mentale Repräsentation der vom Text evozierten Thematik. Heinen (2001) hat das Phänomen des Weltwissens am Zusammenhang von Lesezeit und Verstehen von Texten untersucht. Dabei kommt er zu dem erstaunlichen Ergebnis, dass falsche Sätze innerhalb eines Textes die längste Reaktionszeit hervorrufen, ehe sie vom Rezipienten abgelehnt werden. Der Befund steht kontraintuitiv zu der Hypothese des Abgleiches des propositionalen Gehalts 
einer Äußerung am Maße internalisierten Wissens, derzufolge nämlich die Unverträglichkeit eines propositionalen Gehaltes mit der mentalen Repräsentanz des Themas zu einer schnelleren Ablehnung der betreffenden Äußerung führen sollte, da eine quasi objektive Inkompatibilität die Bewertung der Proposition als falsch erleichtern müsste (siehe Heinen 2001: 128). Hier ist zusätzlich der in Heinens Arbeit nicht diskutierte Begriff des «Textsinnes» als Erkenntnis des «Grund des Geäußertseins» (dazu Daiber 2014 i. Dr.) eines Kommunikates heranzuziehen. Kommunikate sind invariant mit einer bestimmten Intentionen ihrer Produktion verbunden, und die Herstellung einer mentalen Repräsentation der mutmaßlichen Intentionen eines Sprechers erfordert sicher einen höheren kognitiven Aufwand als die Abgleichung propositionaler Gehalte. Beides, nämlich die Wichtigkeit der Intention und eine gewisse Rezeptionsverzögerung, lässt sich mittels indirekter Sprechakte und unter diesen prominent mittels ironischer Äußerungen hinreichend belegen. Das Verständnis einer ironischen Äußerung ist wesentlich daran geknüpft ist, dass ein Rezipient erstens die sachliche Unangemessenheit eines propositionalen Gehaltes im Vergleich mit der mentalen Repräsentation des Sachverhaltes erkennt und zweitens die Intention versteht, die der Produktion der in der Regel hyperbolischen Proposition zugrunde liegt. Die Fähigkeit, die mit einem Kommunikat verbundenen Intentionen zu erschließen, wird in der «Theory of Mind» modelliert, wobei die Ergebnisse den erwarteten Zusammenhang zwischen Ausbildung eines Selbstbewusstseins und der Fähigkeit, das «Selbst» des sprachlichen Gegenübers analogisch zu erschließen, bestätigen (Badstieber 2008). Für den Begriff des kommunikativen Gedächtnisses bedeutet dies, dass die Gesprächspartner in der Kommunikation nicht nur die markierte (d. h. kommunikationswürdige) Einordnung von Geschehnissen in einen Rahmen aushandeln, sondern die Kommunikation auch auf dem gemeinsamen Wissen von relativ zu einem gegebenen Thema intentional erwartbaren Propositionen beruht. Wird ein unerwarteter Aspekt des Themas angesprochen, wird dies als intentionale Akzentsetzung des Sprechers verstanden. Nur unter Berücksichtigung des Begriffes der Intention ist eine psychologische Auswertung biographischer Narrative überhaupt gerechtfertigt, und auch die Gesprächslinguistik hat unter Berücksichtigung des Zusammenspiels von «Rahmen» und «Intention» eine methodische Prämisse: Alles, was gesagt wird, darf als intentional Gesagtes gedeutet werden, was im irreversibel linearen Verlauf der mündlichen Medialität bedeutet, dass auch die Folge der einzelnen Äußerungen als bedeutend interpretiert werden darf. Nur deshalb ist die Rede von einer "Textstrategie» in der medialen Mündlichkeit mehr als metaphorisch. Da anders als in der medialen Schriftlichkeit dem Produzenten spontanes Handeln abverlangt wird und keine Möglichkeit zur Redaktion der einmal getätigten Äußerung bleibt, bedeutet «Strategie» bei der Gesprächsanalyse den Nachvollzug der vom Textproduzenten hergestellten intentionalen Ordnung eines Themengebietes. 


\section{Methodik der Analyse}

In dem Konzept der «intentionalen Ordnung eines Themengebietes» scheint mir die Vorstellung eines sich intersubjektiv angleichenden kommunikativen Gedächtnisses mit der allgemeinen Form mündlichen Redens am besten vereinbar: Die Kommunizierung von Gedächtnisinhalten ist intersubjektiv aufgrund der Referenz auf gemeinsames Weltwissen und auch intersubjektiv im Einverständnis darüber, was nicht für intentional markiert erklärt werden muss. Die Intersubjektivität von Intentionen kann sich zwar einerseits verbal vollziehen, indem sich Produzent und Rezipient explizit über Absichten austauschen, vollzieht sich aber elementarer dort, wo der «Grund des Geäußertseins» des Kommunikates oder einzelner Propositionen nicht der Verständnissicherung (etwa in Parenthesen; vgl. zu der kognitiven Funktion «textuell defokussierter Handlungsmuster» Lampert 1992: 136) bedarf.

Indem Gesprächslinguistik nicht nur auf die lineare Abfolge der Propositionen und ihre gegebenenfalls verständnissichernde Einordnung in einen «Rahmen» zu achten hat, sondern darüber hinaus auch das Nichtverbalisierte des Kommunikates berücksichtigen muss, ergibt sich ein methodisches Problem. Was überhaupt ist an einem Produkt der medialen Mündlichkeit nicht interpretationsfähig? Auf der einen Seite lässt sich die Interpretation von Transkripten nach Maßgabe des Vorstehenden einschränken auf die Kriterien: Reihenfolge der Propositionen relativ zu einem Thema und die Analyse evaluierender oder diskursiv-interaktiver Markierung. Auf der anderen Seite wird ein grundsätzlicher «Zeugnischarakter» mündlicher Medialität bemerkte, welcher vor allem auf der von Transkripten schwer einholbaren intonatorisch-artikulatorischen Ebene angesiedelt ist:

„mowa żywa jest świadectwem pewnego sposobu doświadczania świata i jego konceptualizacji, a w subtelnych i bogatych środkach prozodycznych, którymi operuje każdy mówiący (nie może ich nie stosować) wypowiedzi mówione przenoszą cały system wartości i ocen. Dlatego ustne przekazy mają walor świadectwa" (Bartmiński, Niebrzegowska-Bartmińska 2009: 82).

Der von den Autoren festgestellte Zeugnischarakter der mündlichen Rede ergibt sich wesentlich aus dem multi-modalen Zusammenspiel von Verbalität und Ikonizität bzw. Indexikalität. Während Verbalität das stilistische Register der «Nähesprache» (vgl. den klassischen Aufsatz von Koch, Österreicher 1985) bezeichnet, wo sämtliche diskursiv-pragmatischen Mittel im weitesten Sinne anzuführen wären, treten ikonische Mittel im Bereich von Intonation bzw. Artikulation, aber auch im Bereich sprechbegleitender Gesten auf, und indexikalische Mittel nochmals im Bereich von Artikulation (etwa: Redepausen als Anzeichen von Nachdenklichkeit) und kommunikativem Verhalten (etwa: Proximität der Körper, Augenkontakt). Je eindringlicher sämtliche Umstände der Kommunikationssituation als das Kommunikat begleitend oder relativierend untersucht werden, umso wichtiger scheint jedes Detail zum Verständnis des Kommunikates zu werden. 
In welchem Maße sind nonverbale Umstände der Kommunikation als Teil des Kommunikates zu berücksichtigen? Die von Bartmiński und Bartmińska (2009) angesprochene lautliche Seite der mündlichen Medialität kann in Transkripten durch entsprechende Zusatzzeichen in unterschiedlicher Detaillierung (Selting et al. 2009) erfasst werden. Darüber hinaus aber fragt sich, ob mündliche Medialität angemessen ohne Rücksicht auf visuelle Begleiterscheinungen aus den Bereichen Geste, Mimik und Körperhaltung zu verstehen sei? Unter dem Gesichtspunkt, dass redebegleitende Körperbewegungen den propositionalen Gehalt von Worten nicht nur betonen, sondern auch ikonisch spezifizieren oder mit ihm kontrastieren können, weist Enfield (2009) vor allem auf deiktische und illustrierende Gesten hin, während Rossini (2009: 225) auch von einer speziellen Semantik redet: «coverbal gestures also show a high degree of abstraction, especially if performed during spontaneous restatement». Während redebegleitende Gesten gewisse abstrakte Merkmale des Redethemas hervorheben und so fokussieren können, ist allerdings klar, dass nur sehr wenige Gesten als konventionalisierte Zeichen und damit nicht als redebegleitend, sondern als redekonstituierend zu betrachten sind, wie etwa (kulturspezifisch) Kopfnicken als Bejahung oder deiktisches Anzeigen eines Gegenstandes anstelle einer verbalen Aussage über seinen Aufenthaltsort. Während die redebegleitenden Gesten semantisch das lexikalische Konzept oder die Modalität der Proposition spezifizieren können und redekonstituierende Gesten als Ersatzhandlungen für Sprechakte eintreten, muss aber bemerkt werden, dass Gesten nie thematisch, sondern immer nur rhematisch auftreten. Gesten können kein Textthema einführen, sondern treten nur dort auf, wo der Grund des Geäußertseins der Textthematik schon bekannt ist; redebegleitende Gesten verstärken die rhematischen Teile der einzelnen Propositionen, redekonstituierende Gesten aber sind als Redeersatz nur deshalb verständlich, weil die entsprechende verbale Handlung, für welche sie eintreten, den Gesprächspartnern rekonstruierbar ist. Insofern ist Gesprächslinguistik, welche die lineare Anordnung von Propositionen relativ zu einem gegebenen Thema interpretiert, auf die Berücksichtigung redebegleitender und redekonstituierender Gesten in den Transkripten angewiesen, jedoch ist der Vermerk gestischen Handelns im Falle redebegleitender Gesten immer bereits eine Frage der Interpretation (Akyol 2003: 12 spricht von «transparenten, halbtransparenten und nicht-transparenten» Gebärden). Während das Phänomen Redepause in Transkripten durch die physikalische Messung der Dauer objektiv angegeben werden kann, während auch artikulatorisch-intonatorische Phänomene sich phonographisch objektivieren lassen, ist der Vermerk von Gesten in Transkripten bereits ein Zeichen dafür, dass der Rezipient hier eine Körperbewegung für verständnisrelevant erachtete, was dem Charakter von Transkripten, nämlich möglichst objektives Rohmaterial für die Interpretation zu sein, in gewissem Sinne zuwiderläuft.

Von den als redebegleitend oder redekonstituierend empfundenen Gesten sind die Phänomene der Körpersprache m. E. zu unterscheiden. Indem das Redeverhal- 
ten von Menschen zugleich Produkt und Medium ihrer Sozialisation ist, bemerken Gesprächspartner, die eine vergleichbare Sozialisierung durchlaufen haben, aneinander Verhaltensabweichungen, die als Einschränkung der Glaubwürdigkeit der verbalen Äußerung des Anderen interpretiert werden. Die interkulturelle Problematik, die sich an den Begriff der Sozialisierung hängt, wäre hier genauer darzustellen, aber wir konzentrieren uns hier auf das methodische Problem.

Der von Bartmiński und Bartmińska (2009) herausgestellte «Zeugnischarakter» der mündlichen Gesprächssituation zielt auf ein zentrales Gebiet der mit jedem Sprechakt verbundenen Intention des Sprechenden: auf die Frage nach der Authentizität bzw. Glaubwürdigkeit. Während literaturwissenschaftliche Arbeiten die Frage nach der Glaubwürdigkeit im Kontinuum zwischen Fiktionalität und Historizität ansiedeln, wird das Problem der Glaubwürdigkeit linguistisch aktuell anhand von Internettexten besprochen, die zumeist anonym veröffentlicht werden, was den Rezipienten allerdings keine Einschränkung der Glaubwürdigkeit signalisiert (Chesney, Su 2010). Ebenso wie das Rezeptionsverhalten im Internet medienspezifisch ist, ist medienspezifisch auch eine gewisse Aufmerksamkeit der Gesprächspartner auf das Redeverhalten des Gegenübers in der mündlichen Medialität, besonders, wenn die Gesprächspartner sich gegenseitig materielle Interessen unterstellen können wie etwa in Verhandlungen oder Verkaufsgesprächen. Weil sich eine wahrheitsabweichende Aussage in der mündlichen Gesprächssituation oftmals schneller, nämlich schon beim Aussprechen der Äußerung als solche entlarvt, werden Polizeiverhöre nach Vorladung durchgeführt oder wird bei Gerichtsverhandlungen das persönliche Erscheinen der Zeugen angeordnet.

Auch die Einschätzung von nonverbalen redebegleitenden oder - in Ausnahmefällen - redekonstituierenden Körperbewegungen (von Geste bis Proximität) beruht auf der Erkenntnis der Intentionalität des Kommunikates, d.h. auf seiner Interpretation. Wie sich nun der propositionale Gehalt eines affirmativen Satzes S1 nicht durch den Vordersatz «Es ist wahr, dass S1» ändert, so ändern nonverbale Glaubwürdigkeitssignale auch nichts an dem propositionalen Gehalt der verbalen Äußerung. Alleine die «verräterischen» Gesten bilden eine spezielle Klasse, indem sie die vorausgesetzte Glaubwürdigkeit des Gesprächspartners beim Rezipienten irritierend in Frage stellen.

Die bisherigen Überlegungen zusammenfassend besteht die Methodik der Gesprächsanalyse mittels Transkripten zur Aufdeckung der Mechanismen des kommunikativen Gedächtnisses darin, die thematisch setzenden und möglicherweise textsortenspezifischen Propositionen zu erkennen, redekonstitutive nonverbale Elemente zu bemerken und die Linearität der einzelnen Propositionen als intendierte zu interpretieren, also die Ordnung von der themensetzenden ersten Proposition bis zur das Thema beschließenden Evaluation darzustellen, wobei nonverbale Elemente als rhematisch-modale Spezifikationen wie auch nonverbale Glaubwürdigkeitssignale außer Acht bleiben, da sie nicht dem Bereich der Textplanung zugehören. Die Aufgabe der Analyse umfasst auch, in der textplane- 
rischen Strategie jene Momente herauszuheben, in welchen die Gesprächspartner den propositionalen Gehalt des Gesagten für kommentarwürdig erachten bzw. was dieselbe Frage ist - jene Momente auszuzeichnen, wo vielmehr eine Hervorhebung des propositionalen Gehaltes der Äußerung für überflüssig erachtet wird: die intersubjektive Gestalt des kommunikativen Gedächtnisses bildet sich im Widerspiel von «Normalität», also nicht-kommentierungswürdigem propositionalen Gehalt, und individueller Motivierung ab.

\section{Fallbeispiel}

Das folgende Transkript als Anfangsteil eines Interviews besitzt einen halböffentlichen Charakter, indem die Gesprächspartnerinnen verabreden, ab sofort das Mikrophon einzuschalten und so quasi mit der anonymen Öffentlichkeit bereits rechnen. Die vorformulierte Frage lautete: «Was hat Dich dazu bewogen, Lehrerin zu werden?»

1 (.) эмм (---)

2 я всегда интересовалась иностранными языками

3 даже когда

4 (.) эм (.)

5 после окончания музыкальной школы, после девятого класса

$6 \quad$ (.) эмм (.)

7 хотела поступать в музыкальный колледж

8 (---) э (---)

9 всё-таки изменила свое решение

10 и:::

11 хотела продолжать дальше учиться в школе

12 чтобы поступить потом в педагогический университет

13 и (.) изучать инностарнные языки.

14 (.) эм

15 после окончания университета

16 (.) эм

17 я (.) очутилась в школе

18 естественно чтоб попробовать себя (--) м педагогом

19 и:::

сразу же после первых, можно сказать, двух-трёх, (.) четырёх месяцев

21 я увидела, что эта профессия (.) доставляет мне удовольствие

22 (.) эм (.) 


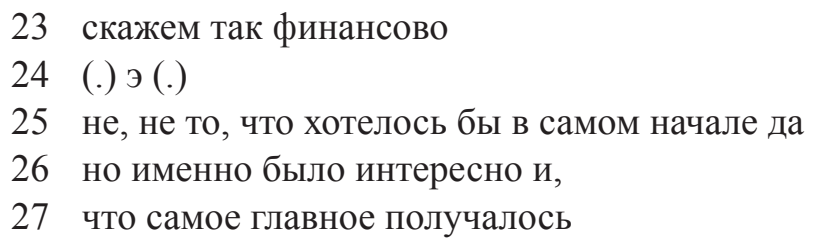

Nach einer Pause [1], die dazu dient, einen Anfang zu dem gegebenen Thema «Lehrerin» zu finden, stellt die Sprecherin das Thema allgemein dar [«immer», 2], um dann die Folie einschränkend [«sogar», 3] zu kontrastieren. Dieser Verlauf ist ein typisches Narrationsschema, den erzählenswürdig ist immer nur das Außergewöhnliche, also jenes, was sich vor der Normalität («immer») heraushebt. In diesem Falle ist die Überleitung in das spezifisch zu Erzählende allerdings gestört. Davon zeugen schon die zwei längeren Pause [4, 6], welche die Formulierung des Spezifikums begleiten. Der propositionale Gehalt $[5,7]$ der schließlich gefundenen Äußerungen widerspricht dann der allgemeinen Themendarlegung, denn obgleich sich die Erzählerin «immer» für Sprachen interessiert habe, hat sie sich doch nach dem Ende der allgemeinbildenden Schulzeit an einer Musikschule («9. Klasse») für die Oberstufe in einem «College» entschieden. «Trotzdem» [9] habe sie dann ihre Entscheidung geändert.

Die Propositionen [1] bis [9] sind von einem Missverhältnis zwischen den kohäsiven Mitteln, welche die Rede strukturieren, und dem propositionalen Gehalt der Sätze gekennzeichnet. Nicht nur widerstreitet das behauptete «immer» [2] der Sprachbegeisterung mit der Entscheidung für die Musik [7], sondern auch der Satzabbruch nach [3], zu erkennen an der Rephrasierung des Temporaladverbs «als» durch die Temporalphrase «nach Beendigung der Musikschule», deutet an, dass der Sprecherin Gründe für die Entscheidung zum Lehrerberuf bewusst sind, die nicht verbalisiert werden, weshalb auch die Entscheidung ein kommentarloses «trotzdem» bleibt. Während die kohäsiv gliedernden Mittel der Rede [3, 9] eine logische Verknüpfung der Propositionen signalisieren, als ob es einschränkende Kriterien für das «immer» [2] der Berufswahl gebe, wird diese logische Gliederung semantisch nicht erfüllt. Nach nochmaliger längerer Pause [10] findet die Sprecherin dann flüssig zum Abschluss des ersten Themenblockes, dass sie nämlich «weiter lernen wollte» [11], und zwar auf einer «pädagogischen Universität» [12] «Fremdsprachen» [13]. Offenbar, wie die Bezeichnungen der verschiedenen Lehranstalten andeuten, handelte es sich darum, dass sich die Sprecherin für die gymnasiale Oberstufe der nun nur noch als «Schule» bezeichneten «Musikschule» entschied, um nicht einen musikalischen Abschluss auf einem «College» - vielleicht einer Privatschule - anzustreben, sondern die Universitätsreife zu erlangen.

Proposition [15] nimmt [5] wörtlich wieder auf («nach Beendigung») und spricht von der Referendariatszeit, wobei wieder ein gewisser Widerspruch sichtbar wird zwischen der Formulierung «geriet auf eine Schule» [17] und der 
stilistisch sehr formalisierten Berufsbezeichnung «Pädagoge» [18]. Die Wortfindungspause vor «Pädagoge» und die nachfolgende Pause [19] stehen in Widerspruch zu der kohäsiv angezeigten temporalen Ordnung der Adverbien [2, 5, $15,20]$, denn es wird keine flüssige Narration produziert. In [20] kontrastiert das Adverb «sofort» mit der Zahlenangabe der Monate, welche die Eingewöhnung an der Schule gebraucht habe. Die Zahlenangabe «zwei, drei Monate» wäre idiomatisch als ikonische Nachahmung einer kleinen Menge zu verstehen gewesen, der Zusatz «vier» hebt das Idiomatische der Wendung auf und zeigt, dass die Eingewöhnungsphase doch länger war, als die «normale» idiomatisch ausdrückbare Zeit und widerspricht damit dem «sofort», mit dem der Satz eingeleitet ist. Ebenso wie die «immer» vorhandene Begeisterung für Fremdsprachen ist auch der «sofortige» Erweis der Berufseignung nicht ganz glaubhaft. Die formelhafte - wie zuvor schon die «Pädagogin» [18] - das unpersönliche, konzeptionell schriftliche Register anzielende erste Evaluation, «der Beruf bereite Befriedigung» [21], lässt den Hörer wie das unerklärte «trotzdem» [9] über die individuellen Motive und die emotionale Beteiligung der Sprechenden im Unklaren. Der unpersönliche, allgemeine Gehalt in [21] hätte als Evaluierung des gesamten Geschehens das Thema abschließen können, aber die Angabe der «Befriedigung» wird nun doch spezifiziert, dass sie nämlich nicht «finanzieller» [23] Art sei, wie das wohl «im Anfang» [25] angenommen wurde, aber «es» (der Beruf? die Ausbildung?) «war» (!) interessant [26] und «was die Hauptsache sei» habe die Sprecherin auf ihren Berufsweg gebracht [27].

Es ist unschwer zu sehen, dass in dem kleinen Textausschnitt eine vollständige Narration vorliegt, welche temporale und logische Orientierung der einzelnen Propositionen signalisiert und in einer evaluierenden («es hat funktioniert») Betrachtung zu Ende gebracht wird. Es ist weiters offenbar, dass die kohäsiven Mittel der Redegliederung und die Kohärenz der Äußerungen auseinanderfallen: man versteht nicht, warum das immerwährende Interesse an Sprachen den Umweg über die Musik nötig machte, man versteht nicht, warum die «Selbsterprobung» [18] so wenig initiativ [17] und so wenig persönlich [18: «Pädagoge»] versprachlicht ist. Das appellative «sagen wir mal» [23], welches auf das Thema der Entlohnung überleitet, darf man als Aufforderung an die Hörerin verstehen, auch für die materiellen Gründe der Berufswahl Verständnis aufzubringen. Die ohne thematischen Zwang nachgestellte finanzielle Begründung lässt sich erkennen als Grund für den unerklärt gebliebenen Wechsel von der Musik zu den Sprachen und für die unpersönliche Art der Selbstbeschreibung: der Beruf wurde auch aus Vernunftgründen, nicht nur aus Neigung gewählt.

Nicht nur das Gesagte in [1] bis [21] stellt sich im Lichte von [22] bis [25] neu dar, sondern auch das Ungesagte. Wir können die ungesagten Gründe für die Berufswahl erraten, während die verbalisierten Äußerungen sich als unpersönlich und floskelhaft erweisen. Im Nachvollzug der Entsprechung kohäsiver Signale mit dem geäußerten propositionalen Gehalt ergibt sich die Kohärenz auch die- 
ses Textes, wobei die Linearität der Abschnitte von einem öffentlichen zu einem privaten Teil leitet. Der öffentliche Teil produziert im Zeitalter der «Selbstverwirklichung» ein Selbstbild des Individuums, das seine berufliche Tätigkeit nach Begabung und Begeisterung wählt; der linear nachgeordnete privatere Teil kann die geringer geschätzte finanzielle Motivation erwähnen. Es ist natürlich nicht sachgerecht, die Sprecherin nun für «überführt» zu halten und ihr «verborgene», aber vom Interpreten nun «freigelegte» Intentionen nachzuweisen. Vielmehr ist der Text ein Musterbeispiel für das Funktionieren des kommunikativen Gedächtnisses, indem die Sprecherinnen in einem halb-öffentlichen Gespräch sich auf öffentliche, allgemein akzeptierte Handlungsintentionen verständigen, und sowohl im verständnisheischenden скажем, wie in der linearen Nachsetzung des weniger öffentlich akzeptierten Handlungsmotives genau die Wertmaßstäbe der Öffentlichkeit reproduzieren. Ohne auch nur am Rande gesellschaftliche Rahmenbedingungen zu verbalisieren, performiert der Textausschnitt eine gesellschaftliche Ordnung, in der «Neigung» [2: всегда] und «Entscheidung» [9: решение] zur «Selbstverwirklichung» [18: попробовать себя] in einer anerkannten Tätigkeit [18: педагог] und persönlicher Befriedigung [21: удовольствие] zu konvergieren haben, während die allen [23: скажем] bewussten niederen Motive erwähnt, aber nicht für ausschlaggebend erachtet werden können. Ausschlaggebend ist vielmehr das «Interessante» [26], dessen Präteritum entweder als Anzeige der erzählten Zeit gelten kann oder - wofür die Stellung in der evaluierenden Position spricht für eine gewisse resignierende Bewertung zum Erzählzeitpunkt.

\section{Das erinnerte Kommunikat}

Die in der medialen Mündlichkeit genau eine Minute dauernde Narration der Sprecherin ist für deren erste Rezipientin unmöglich in derselben Detailliertheit wie hier zu verstehen. Die Nichtäquivalenz von kohäsiven Gliederungssignalen und kohärenten Propositionen wird nur beim Lesen des Transkriptes einsichtig. An was erinnern sich nun die Kommunikanten, was bildet den Inhalt des Kommunikates? Weder wird hier behauptet, dass die Sprecherin «unbewusst» über den Einwand des Finanziellen in ihrer Textplanung stolperte und daher Kohäsion und Kohärenz auseinanderfallen, noch wird behauptet, dass die Rezipientin «unbewusst» die Wichtigkeit der finanziellen Botschaft doch gehört habe. Es ist genauso möglich, dass die Sprecherin bewusst das finanzielle Thema zunächst zu umgehend suchte, da es ihr in der Linearität der Erzählung am Anfang nicht passend erschien, und es ist genauso möglich, dass das Hinauslaufen der Rede auf das finanzielle Thema von der Hörerin als wichtiger Punkt, der die Evaluierung der Narration möglich machte, verstanden wurde. Wenn von Unbewusstem hier die Rede sein könnte, dann nur in dem Sinne der Verinnerlichung, der Anwendung von sprachlichen Verhaltensmustern, in diesem Falle Erzählschemata, welche 
auch eine gewisse Hierarchie des am Anfang und des am Ende Erwähnbaren enthalten. Der «Rahmen Berufswahl» ist intersubjektiv in seinen wichtigen Ablaufstationen (Ausbildung, Prüfung, Berufserfahrung) bekannt und die Propositionen werden ihm kohärent eingeordnet. In der expliziten Evaluierung verständigen sich die Subjekte über die öffentliche Anerkennung von Ereignissen («Pädagoge»), in der formalen Darlegung des Sachverhaltens darüber hinaus auch über die Rangfolge von Werten («Neigung» vor «Geld»). Beides zusammen macht die kleine Alltagserzählung zum kommunikativen Ereignis: Nicht nur die Strukturierung von Erinnerungsinhalten in der Linie öffentlich vertretbarer Bewertung verleiht der Narration den Charakter des kommunikativen Gedächtnisses, sondern auch der Appell an das Verständnis nicht explizit verbalisierter Motive, dass nämlich Geld von Anfang an eine wichtige Überlegung bei der Berufswahl bildete. Die Linearität der Themenfolge performiert eine Rangfolge von Werten.

Am Ende möchte ich noch darauf hinweisen, dass die kleine Analyse keine Notwenigkeit hervorruft, das «kommunikative Gedächtnis» entweder auf psychologischer (kapitalistisches Über-Ich?) oder auf sprachsystemischer (языковая картина мира?) Ebene zu behandeln. Die linguistische Gesprächsanalyse kann das Phänomen auf der Ebene der Textpragmatik belassen als durch Sozialisation erworbene Kompetenz sprachlichen Verhaltens. Zwar könnte man dem Gespräch gesellschaftskritische Bemerkungen zum Stellenwert der Arbeit und ihrer diskursiven Darstellung entnehmen, aber es besteht kein Grund, dem Diskursmuster «Talent und Selbstverwirklichung» eine über die mechanische Anwendung hinaus zwingende Kraft zuzuschreiben, die es der Reflexion der Diskursteilnehmer entzöge. Die Interpreten des Diskurses sind als Teilnehmer des Diskurses der erste Beweis für die Möglichkeit, die «Ordnung der Dinge» kritisch ihrer universalen Mächtigkeit zu berauben, wenn - wie etwa im Falle einer Transkription - der Automatismus sozusagen in Zeitlupe rezipiert werden kann. Außerdem ermöglichen erlernte Diskursmuster, auch in jenen Fällen eine Kommunikation aufrechtzuerhalten, wo das Individuum seiner Rede möglichst nicht den Charakter eines «Selbstzeugnisses» geben will. Bei einer ideologiekritischen Diskursanalyse ist daher auch zu fragen, welches neue Diskursmuster als entlastende Normalität angeboten werden soll.

\section{Literatur}

Akyol, S. (2003), Nicht-intrusive Erkennung isolierter Gesten und Gebärden. Diss. Ing.-Wiss. TH Aachen.

Assmann, J. (2000), Körper und Schrift als Gedächtnisspeicher. Vom kommunikativen zum kulturellen Gedächtnis, [In:] M. Csáky, M., P. Stachel (ed.), Speicher des Gedächtnisses. Bibliotheken, Museen, Archive 1: Absage an und Wiederherstellung von Vergangenheit-Kompensation von Geschichtsverlust, Wien, 199-213.

Badstieber M. 2008, Theory of Mind, episodisches Gedächtnis und Sprache: Eine Längsschnittuntersuchung bei 3- bis 4-jährigen Kindern. Diss. rer. nat. Univ. Frankfurt/ Main. 
Bartmiński J., Niebrzegowska-Bartmińska St. (2009), Tekstologia, Warszawa.

Chesney T., Su D. K. S. (2010), The impact of anonymity on weblog credibility. «International Journal of Human-Computer Studies», 68, 710-718.

Daiber Th. (2014 i. Dr.), Gott in den Medien. Zur Kohärenz in der konzeptionellen Mündlichkeit, [In:] Nagórko A. (ed.), Sprachliche Säkularisierung - Semantik und Pragmatik, Tagungsband, Hildesheim, Zürich. (= westost-passagen. Slawistische Forschungen und Texte).

Enfield N. J. (2009), The Anatomy of Meaning. Speech, Gesture, and Composite Utterances, Cambridge. (= Language, culture and cognition 8 )

Heinen S. (2001), Der Einfluss von Vorwissen, Interesse und Arbeitsgedächtniskapazität auf die mentale Repräsentation von Texten, Diss. phil. Univ. Bielefeld.

Koch P., Oesterreicher W. (1985), Sprache der Nähe - Sprache der Distanz. Mündlichkeit und Schriftlichkeit im Spannungsfeld von Sprachtheorie und Sprachgeschichte, «Romanistisches Jahrbuch», 36, 15-43.

Lampert M. (1992), Die parenthetische Konstruktion als textuelle Strategie. Zur kognitiven und kommunikativen Basis einer Grammatischen Kategorie, München. (= Slavistische Beiträge 284)

Rossini, N. (2009), Gestural Abstraction and Restatement: From Iconicity to Metaphor, [In:] A. Esposito, R. Vích (ed.), Cross-Modal Analysis of Speech, Gestures, Gaze and Facial Expressions, Berlin, Heidelberg, 214-226. (= Lecture Notes in Artificial Intelligence 5641)

Schmitt, M. (2009), Gedächtnis als soziologisches Problem, [In:] ibid., Trennen und Verbinden. Soziologische Untersuchungen zur Theorie des Gedächtnisses, Wiesbaden, 21-81.

Selting, M., Auer, P., Barth-Weingarten, D., Bergmann, J., Bergmann, P., Birkner, K., CouperKuhlen. E., Deppermann, A., Gilles, P., Günthner, S., Hartung, M., Kern, F., Mertzlufft, Ch., Meyer, Ch., Morek, M., Oberzaucher, F., Peters, J., Quasthoff, U., Schütte, W., Stukenbrock, A., Uhmann, S. (2009), Gesprächsanalytisches Transkriptionssystem 2 (GAT 2). «Gesprächsforschung - Online-Zeitschrift zur verbalen Interaktion», 10, 353-402.

\section{Томас Дайбер}

\section{Стратегия устных нарративов и коммуникативная память}

\section{Резюме}

Культурологическое понятие «коммуникативной памяти» является вопросом лингвистики текста, демонстрируется на конкретном материале общий всем индивидам вид биографической повести, которая соединяет индивидуальное содержание воспоминаний и общую форму его оценки.

В статье сравнивается характеристика коммуникативной памяти как «пределы (рамы) опыта» с более широким лингвистическим понятием «всем известное знание мира» (commonsense knowledge, Weltwissen), обсуждаются методологические вопросы анализа устных нарративов. На материале короткого, замкнутого устного повествования в статье проиллюстрировано, каким образом собеседники сообщают друг другу общие для них ценности. 